\title{
Organizaciones internacionales de investigación, ensayo y normalización del cemento
}

JOSE MARIA BALAGUER

Asland, S. A.

Ante todo quiero hacer constar que no voy a pronunciar ninguna conferencia. Mi labor será meramente informativa de cómo ciertas Asociaciones Internacionales investigan sobre el cemento principalmente en vistas a posibles ensayos de calidad pero que entrañan un conocimiento profundo (o lo más profundo posible) del cemento y por eso precisa de la investigación.

Todos sabemos que en todas las naciones más o menos industrializadas existen comités de Normalización que indican lo que hay que hacer para saber si un producto que se vende es bueno o malo, es decir, si reúne o no unas condiciones mínimas de aceptación con vistas al público a que va destinado. Nosotros, los cementeros, tenemos buena experiencia de ello y sabemos que no es fácil; pero si pasamos el problema al campo internacional, fácilmente se comprende que las dificultades aumentan en proporción geométrica.

Es indispensable, por tanto, reunirse, hablar, discutir, ensayar de común acuerdo, volverse a reunir, discutir resultados y al fin ponerse de acuerdo intentando salvaguardar al máximo los intereses de nuestras respectivas naciones que, si bien por su propia soberanía no están obligadas a aceptar normas, no cabe la menor duda que cualquiera de ellas aceptada internacionalmente repercute quiérase o no en cada una de las naciones miembros de la organización.

Por ello las recomendaciones e incluso las normas internacionales adolecen de cierta imprecisión por poco taxativas, pues deben tener cierta elasticidad para que el técnico la interprete y adapte a su caso, sin interferir nunca en el soberano derecho de las naciones a decidir lo que crean conveniente sin ingerencias exteriores.

Pero ... existe el comercio de exportación y en infinidad de casos la nación importadora y la exportadora, aunque midan los mismos parámetros las cifras limitativas no son las mismas, por ejemplo: R.I. $\mathrm{SO}_{3}$ - P.F. etc., o varían los métodos de ensayo.

Resulta, por tanto, indispensable una vez más el ponerse de acuerdo, y esto es el propósito del CEN (Comité Europeo de Normas) que actúa con el beneplácito del Mercado Común Europeo, con sede en Bélgica y presidencia belga; a él también pertenecen otras naciones que no están dentro del Mercado Común ... y por el CEN vamos a empezar la información:

El CEN está organizado por todos los países europeos de libre comercio y, por tanto, por todos los miembros del Mercado Común como se ha dicho. Lo componen los Comités de normas de los distintos países, es decir, los miembros Europeos de la ISO (Internatio- 
nal Standard Organization), Organización Mundial para el estudio y aceptación de normas internacionales de toda clase, siendo los Comités Técnicos TC-74 y TC-71 los correspondientes al cemento y hormigón, respectivamente.

Uno de los objetivos del M.C.E. es la unificación de Normas de Calidad entre sus miembros y utiliza al CEN para este propósito, esperando que el M.C.E. obligue a sus socios a aceptar esas normas y, por ello, la industria de los países del M. C. sigue al CEN.

En la cuarta reunión del CEN, los días 22 y 23 de octubre de 1975, en Düsseldorf, quedó estipulado el proceso que debe seguir un ensayo para convertirse en norma CEN. Es como sigue: Cuando un proyecto de propuesta ha sido aprobado por los miembros del Grupo de Trabajo correspondiente (en el caso del cemento sería el CEN/WG-51), el Secretariado del Grupo de Trabajo lo manda al Secretariado General, el cual lo registra, numera y lo manda a todos los organismos del CEN para su conocimiento y estudio.

Este período de encuesta dura seis meses y permite a los Comités de Normas de las distintas naciones, el hacer sus encuestas nacionales y opinar sobre el particular.

Una vez pasado el período de votación, el Secretariado Central junto con el Secretariado del Grupo de Trabajo estudian el resultado de la votación.

Un proyecto de norma Europea se puede considerar aprobado cuando una mayoría substancial de los miembros del CEN ha votado a favor:

- El número de votos a favor será por lo menos el doble del número de votos en contra, sin contar las abstenciones y con la condición de que hayan votado a favor por lo menos cuatro naciones.

- Si un proyecto de norma trata de un punto sobre el cual la Comunidad Europea se propone estandarizarlo en una de sus normas, ésta será considerada como aceptada si cumple con los requisitos antedichos por los votos de los miembros de pleno derecho.

Si se considera que la mayoría obtenida no corresponde como mínimo a los $2 / 3$ de la producción de los países representados en el CEN y a $2 / 3$ del consumo en esos mismos países del producto considerado, un mínimo de dos países puede pedir que no se adopte.

Si no se acepta por el CEN se devuelve al Secretariado del Grupo de Trabajo para que prepare un nuevo proyecto. Este nuevo documento debe pasar por los mismos trámites.

En el caso de que se acepte, la Secretaría Central somete el texto enviado por el Grupo de Trabajo a votación por el Consejo directivo. El período de votación es de dos meses (si bien es discutida todavía su duración).

Después de que el texto ha sido adoptado el Secretariado Central lo hace circular a todos los miembros del CEN. Los miembros que han votado afirmativamente se comprometen a introducir lo acordado en sus normas nacionales, mientras los que hayan votado en contra no están obligados a ello.

No creo necesario hacer hincapié en la importancia que todo esto puede tener para la Industria Cementera de nuestro país en un futuro.

\section{SO}

En la exposición anterior hemos hecho mención de la ISO (International Standard Organization). 
La normalización es una necesidad de la industrialización de los países, que se sirven de un determinado número de productos de una determinada calidad que hay que comprobar por razones obvias.

Se empezó por organismos nacionales y así fue creado primero en Gran Bretaña el BSI (Brithis Standard Institute) en 1901. Muchos países industrializados crearon organismos similares hacia el 1920.

La primera normalización internacional fue de carácter electrotécnico con la "Comisión Electrotécnica Internacional" (CEI) en 1906, seguida por la "Federación Internacional de Asociaciones Nacionales de Normalización" la ISA (International Standards Association) en 1926, visando principalmente la construcción mecánica. Por amenazas de guerra, en 1942, la ISA dejó de funcionar. En 1944 se fundó el Comité de Coordinación de Normalización de las Naciones Unidas (UNSCC), que agrupaba las organizaciones nacionales de 18 países aliados, sucediendo a la ISA.

En octubre de 1946 se reunieron en Londres 64 delegados de 25 países y de sus deliberaciones nació la ISO de ámbito universal.

En la ISO cabe todo lo que pueda normalizarse, desde la denominación de las partículas sub-atómicas, a la resistencia mecánica de un pañuelo de bolsillo.

Esta organización es sumamente útil en el estado actual de la economía mundial, en la que proliferan las sociedades multinacionales que encuentran dificultades a sus productos debido a las diferentes exigencias de cada país en los que actúan.

Una norma internacional es el acuerdo final entre los comités de la ISO y puede ser aplicada tal cual, o bien a través de su incorporación a las normas nacionales de los diferentes países. Hasta finales de 1971 las conclusiones de los trabajos de la ISO se publicaban como recomendaciones. Como consecuencia de una decisión del Consejo de la ISO, en 1970, se publican como Normas Internacionales.

Un proyecto de norma debe pasar por diferentes fases antes de ser considerada como norma internacional. Debe presentarse al Secretariado General, luego someterse a votación de todos los miembros y si obtiene el $75 \%$ de votos favorables se somete al Consejo de la ISO, para su adopción definitiva y su publicación como Norma Internacional.

En general se trabaja por correspondencia y las reuniones no se producen más que en casos de absoluta necesidad.

España es miembro de la ISO a través del Instituto Nacional de Racionalización y Normalización.

\section{R I L E M}

En el Artículo Primero de sus estatutos se lee: Se constituye bajo el nombre de "Reunión Internacional de Laboratorios de Ensayos e Investigación de Materiales y Construcción", por abreviación RILEM, una asociación sin fines lucrativos conforme a los artículos 60 y siguientes del Código Civil Suizo.

La sede de la RILEM está en Ginebra y el Secretariado General en París.

Sus fines son:

1. ${ }^{\circ}$ Intercambio de información y cooperación, referidas a investigaciones experimentales y ensayos sobre la construcción y sus materiales, en curso o en proyecto en los diferentes Laboratorios en los diversos países participantes. 
2. ${ }^{\circ}$ Estudio de métodos de ensayo en vistas a su perfeccionamiento y su unificación.

3. ${ }^{\circ}$ Intercambio de investigadores científicos.

4. Colaboración con asociaciones nacionales o internacionales para la realización de estos objetivos.

5. ${ }^{\circ}$ Organización de coloquios y reuniones especializadas, no periódicas, y de carácter limitado.

\section{MIEMBROS DE LA ASOCIACION}

La Asociación estará formada por miembros Efectivos - Asociados - Titulares - y de Honor.

Miembros Efectivos: Personas directivas de un organismo oficial o privado, de investigación y ensayo de materiales de la construcción.

Miembros Asociados: Los que, sin cumplir las condiciones anteriores, se ocupan de los mismos fines.

Miembros Titulares: Reservado a organismos oficiales o privados de reconocida importancia.

Miembros de Honor: A requerimiento de la Comisión Permanente.

Como puede verse, en la RILEM se busca más la personalidad del investigador que su representatividad.

La RILEM funciona a base de:

- La Comisión Permanente: Compuesta por un delegado y un representante por cada país que participe, los cuales deben ser miembros efectivos.

- El Consejo: Que es el elemento de Gestión, actúa por delegación de la Comisión Permanente, se reúne normalmente 2 veces al año.

- El Secretariado General: Es el órgano ejecutivo de la Comisión Permanente y procede a las publicaciones, periódicas o no, de acuerdo con el Consejo.

- Comisiones Permanentes de Orientación: Para orientar al Consejo y a la Comisión Permanente, se elegirá por ésta un Comité Consultivo que podrá formar pequeñas comisiones para sus fines.

- Comisión Técnica General: Existe una Comisión Técnica General que supervisa las actividades de las Comisiones técnicas e intercambia información y experiencias entre los miembros.

- Grupo de Coordinación: Es el ejecutivo de la Comisión Técnica General y coordina las actividades de las distintas Comisiones Técnicas y Grupos de Trabajo.

- Comisiones Especiales: La Comisión Permanente puede instituir Comisiones Especiales y Grupos de Trabajo para el estudio de problemas específicos. 
La forma de actuar es la siguiente:

Cualquier proposición para la creación de una nueva Comisión o Grupo de Trabajo será comunicada al Comité Consultivo, en contacto con el cual el autor de la proposición deberá preparar:

1. Definición del objeto de la proposición.

2. Programa de trabajo.

3. Lista de posibles miembros.

4. Calendario provisional indicando el tiempo mínimo estimado para la realización del trabajo propuesto.

Todo esto será transmitido a la Comisión Permanente que decidirá la conveniencia o no, de la propuesta.

Como puede verse, todo esto resulta algo complejo, pero en la práctica no hay mayores dificultades, si bien el trabajo resulta bastante lento, cualidad ésta común a todas las organizaciones internacionales.

En España y en el aspecto de que trata esta información hemos trabajado el Dr. Calleja y yo, en el Comité CDC-4, ya desaparecido, que trataba de la durabilidad del hormigón. Este estudio ha vuelto a reconsiderarse y seguirá con sede en el CERILH, en París, actuando el Dr. Calleja como enlace con el CEMBUREAU, del cual hablaré a continuación.

\section{CEM B UREA U}

\section{"Asociación Europea del Cemento".}

Está formada por las Agrupaciones de Fabricantes de Cemento de los países Europeos de libre comercio. Se comprende fácilmente que para nosotros los cementeros es de suma importancia, pues es la única Asociación de la cual formamos parte como tales fabricantes.

Hasta el momento presente ha funcionado bajo el esquema Apéndice I, en el que claramente se ve que las diferentes Agrupaciones de Fabricantes forman una Asamblea General que nombra un Comité Ejecutivo, el cual, a través de sus Miembros Ejecutivos, dirige las oficinas, secretaría, publicaciones, etc. y el Grupo de Coordinación de los distintos Grupos de Trabajo.

Estos Grupos de Trabajo están formados por las distintas personas científicas y técnicas que investigan y trabajan en nuestra industria, nombradas por las distintas Agrupaciones de Fabricantes de los diferentes países que forman el CEMBUREAU.

Cada grupo de trabajo tiene una misión específica, y así tenemos: Hormigón en la Agricultura; Carreteras de Hormigón; Grupo de Estudio de Ensayos del Cemento y Hormigón, etc. Como se comprenderá estos Grupos de Trabajo pueden variar según lo aconsejen las circunstancias.

Están regidos por un Presidente que reúne los trabajos de los Sub-Comités que estudian problemas específicos; se sirve del Secretariado del CEMBUREAU. Cada Sub-Comité tiene asimismo su Presidente que dirige y coordina los trabajos, y redacta y presenta el documento final de la investigación o estudio efectuado. 
Me referiré al último de los citados Grupos de Trabajo, pues es el que corresponde con mi exposición.

El Grupo de "Estudio de Ensayos" está formado por la reunión de distintos Sub-Comités, con una labor específica encomendada a cada uno de ellos, y así tenemos los siguientes: Principios Estadísticos; Ensayos de Resistencias Mecánicas (método ISO-RILEMCEMBUREAU); Calor de Hidratación; Fraguado; Resistencia Química; Composición Mineralógica del Clínker; Análisis del Hormigón fresco; etc.

Estos Sub-Comités están formados por los representantes de los distintos países interesados en uno o varios de los problemas mencionados, y así tenemos que un mismo delegado puede formar parte de uno o más Sub-Comités según sus preferencias, especialidad y disponibilidad de tiempo y medios adecuados.

Una vez terminado el estudio se redacta el informe correspondiente y se pasa a la Secretaría, que, a su vez, lo comunica al Comité Ejecutivo, el cual decide el ámbito de difusión del estudio y su interés o no en elevarlo a la RILEM o bien a la ISO si se considera que puede constituir una Norma Internacional, si el asunto lo merece.

Pero para aclarar más las cosas, y además porque creo que es mi deber el informar sobre nuestra actuación en el Grupo de Trabajo del CEMBUREAU de Ensayos de Cemento y Hormigón, me voy a referir a la investigación llevada a cabo por un grupo de naciones, entre ellas España, y que me cupo el honor de presidir; estos trabajos pueden servir de ejemplo de cómo van las cosas, aunque no precisamente por su ejemplaridad.

En 1961, en la reunión del Grupo de Trabajo de Ensayos, se propuso la creación de un Sub-Comité con el fin de estudiar si se podía conseguir un método rápido para determinar la resistencia química de un hormigón al ataque por los sulfatos. Fueron varias las naciones interesadas y se propuso a España que aceptara la presidencia, ya que nuestro país está seriamente afectado por este tipo de ataque químico en varias de sus zonas geográficas.

Acepté el encargo, sabiendo de antemano que la labor sería ardua, si no imposible, pero había que comprobarlo.

Se formó este Sub-Comité, tomando parte en él: Alemania, Austria, Bélgica, Gran Bretaña y España; más adelante se incorporaron Holanda y Francia.

Todos los trabajos fueron llevados a cabo to mejor posible, pero no siempre con la rapidez que hubiéramos deseado, debido a la enorme cantidad de trabajo a efectuar en los laboratorios.

La investigación duró hasta 1972 ; y los años 1973-74 y 75 sirvieron para poner a punto el documento que les ha sido entregado y que comentaré más adelante.

No voy a leer año por año la relación de los trabajos efectuados pues serán publicados en el apéndice de esta comunicación, pero sí un resumen de ellos.

Se empezó por hacer la relación de los métodos existentes, comprobando que ninguno de ellos era preceptivo. Viéndose una clara distinción entre los dos aspectos de la resistencia al ataque, una intrínseca por propia naturaleza del cemento y la otra por impermeabilidad del hormigón, así como se hacía notar las diferencias de ataque no sólo por el anión $\mathrm{SO}_{4}{ }^{2-}$, sino también por los cationes $\mathrm{Ca}^{2+}, \mathrm{Mg}^{2+}, \mathrm{Na}^{+}$, etc.

Se redactaron los métodos de ensayo escrupulosamente y nos comprometimos a efectuarlos con la misma escrupulosidad sin introducir la más leve variación.

Con ello nos damos cuenta de la gran dispersión de resultados, pero en medio de ello los métodos KOCH-STEINEGGER y MERRIMAN-GARCIA DE PAREDES, es decir con la modificación de éste último efectuado en el IETcc por el investigador García de Pa- 
redes, eran los dos que menos discrepancias manifestaban y se decidió actuar sobre esos dos métodos.

Se hicieron ensayos en común con los mismos cementos suministrados por las distintas naciones; la arena fue suministrada por Bélgica, que posee la reserva-patrón para el ensayo de resistencias ISO-RILEM-CEMBUREAU.

Una vez recopilados los datos, la concordancia resultó ser suficientemente buena, pero la clasificación con respecto a su resistencia química de los diferentes cementos empleados resultó ser contraria a la experiencia real, es decir, no el cemento más resistente en los ensayos era el más resistente en la práctica y viceversa.

Este, en realidar fracaso, nos demostró la falta de confianza que debíamos tener en los ensayos de laboratorio hasta ahora conocidos y la necesidad de profundizar más en el conocimiento básico de la hidratación de los aluminatos cálcicos del cemento portland y ver si por ello se podría prever otro tipo de ensayo en un futuro.

Pero no podíamos dejar al usuario del cemento sin una solución a sus problemas y por eso se decidió redactar un "Código de Buena Práctica", basado no sólo en las normas de cada país, sino también en la propia experiencia personal de cada uno de los componentes del Sub-Comité.

A principios de este año en este Instituto se efectuó la última reunión del Comité CDC-4 (Concrete Durability Committee) de la RILEMI, a la cual se habían comunicado nuestras experiencias, acordándose la suspensión de sus actividades hasta tener mayores conocimientos básicos a lo que corresponde la nueva organización de estudios en el CERILH que he mencionado anteriormente.

Como se comprenderá, en el CEMBUREAU no podía ocurrir de distinta manera y en el mes de junio del corriente año en la reunión plenaria del Grupo de Trabajo de Ensayos se presentó el documento entregado a Vds.

Pero ¿qué importancia puede tener? es seguro que cualquiera con experiencia en hormigones y ante un posible ataque por sulfatos llegaría a conclusiones y adoptaría soluciones análogas a las descritas; entonces ¿qué significa?

A mi manera de ver es lo siguiente:

1) El reconocimiento universal (la RILEM es de ámbito mundial) de que no existe ningún método de ensayo con suficiente base científica y práctica que pueda ser considerado apto para ser preceptivo.

2) La necesidad de una mayor investigación básica sobre la hidratación del cemento.

3) El acuerdo entre todas las naciones Europeas de libre comercio de que las normas recomendadas en el documento adjunto son las más apropiadas para producir un hormigón lo más resistente posible a los ataques por el ion $\mathrm{SO}_{4}{ }^{2-}$.

Como habrán observado, cualquier decisión toma tiempo y tiempo; todo el mundo es muy susceptible, parece como si el honor Nacional dependiera de la granulometría de una arena o cosa semejante y a menudo hace falta más diplomacia que ciencia para desatascar el carro, pero al fin se consigue.

No sería justo que no hiciera constar aquí mi profundo agradecimiento al Instituto Eduardo Torroja y a todos sus componentes por el apoyo y decidida colaboración que me han prestado, aún antes de que un destacado miembro de él, el Dr. Calleja, fuera nombrado oficialmente también representante de nuestra Agrupación en el mencionado Grupo de Trabajo.

Pero las actuales circunstancias no son las mismas, el ambiente internacional ha cambiado y se ha retraído en ciertos aspectos de la investigación; hay que ir más aprisa. 
Como bien decía nuestro Presidente M. Dutron, el trabajo en el CEMBUREAU es el trabajo de los fines de semana que, si bien es muy de agradecer, es causa de lentitud; hay que reorganizarse y por eso en estos momentos está prácticamente en suspenso la actividad del CEMBUREAU a partir del Grupo de Coordinación el cual propone la modificación expresada en el Cuadro Anexo II.

El Grupo de Coordinación opina que las actividades del CEMBUREAU en los campos de promoción y técnicos, deberían estar divididas en:

— actividades externas (desarrollo del mercado, investigación de mercados, etc.);

- problemas internos relativos a la propia industria del cemento (métodos de ensayo, investigación sobre calidad, etc.).

Por este motivo se propone reemplazar en el transcurso del tiempo los seis Grupos de Trabajo por dos Comités Permanentes:

- uno para el desarrollo y promoción de mercados;

- otro para asuntos de competencia interna de la industria del cemento.

Los Comités Permanentes pueden establecer, en caso necesario, Comités específicos de expertos para trabajos concretos, normalmente limitados en cuanto a su ámbito y duración.

Todos los miembros tendrán el derecho de estar representados en los Comités Permanentes, pero los Comités Específicos estarán limitados estrictamente a expertos, incluyendo, si fuera necesario, expertos que no provengan de la industria del cemento.

Los Comités Permanentes se reunirán normalmente una vez al año y los representantes de los miembros podrán ir acompañados de especialistas para tratar de cualquier tema específico a discutir en la reunión.

Esta propuesta de reorganización se presentará a la Asamblea General de 1977, por lo que la nueva organización lógicamente no podrá funcionar hasta 1978; estamos, pues, a la espera. 


\section{A PEN DICES}

I ORGANIZACION ANTERIOR DEL CEMBUREAU

II PROPUESTA DE REORGANIZACION DEL CEMBUREAU

III RELACION DE TRABAJOS EFECTUADOS POR EL SUB-COMITE DE RESISTENCIA QUIMICA DEL CEMBUREAU ENTRE 1962 A 1976

IV RECOMENDACIONES 


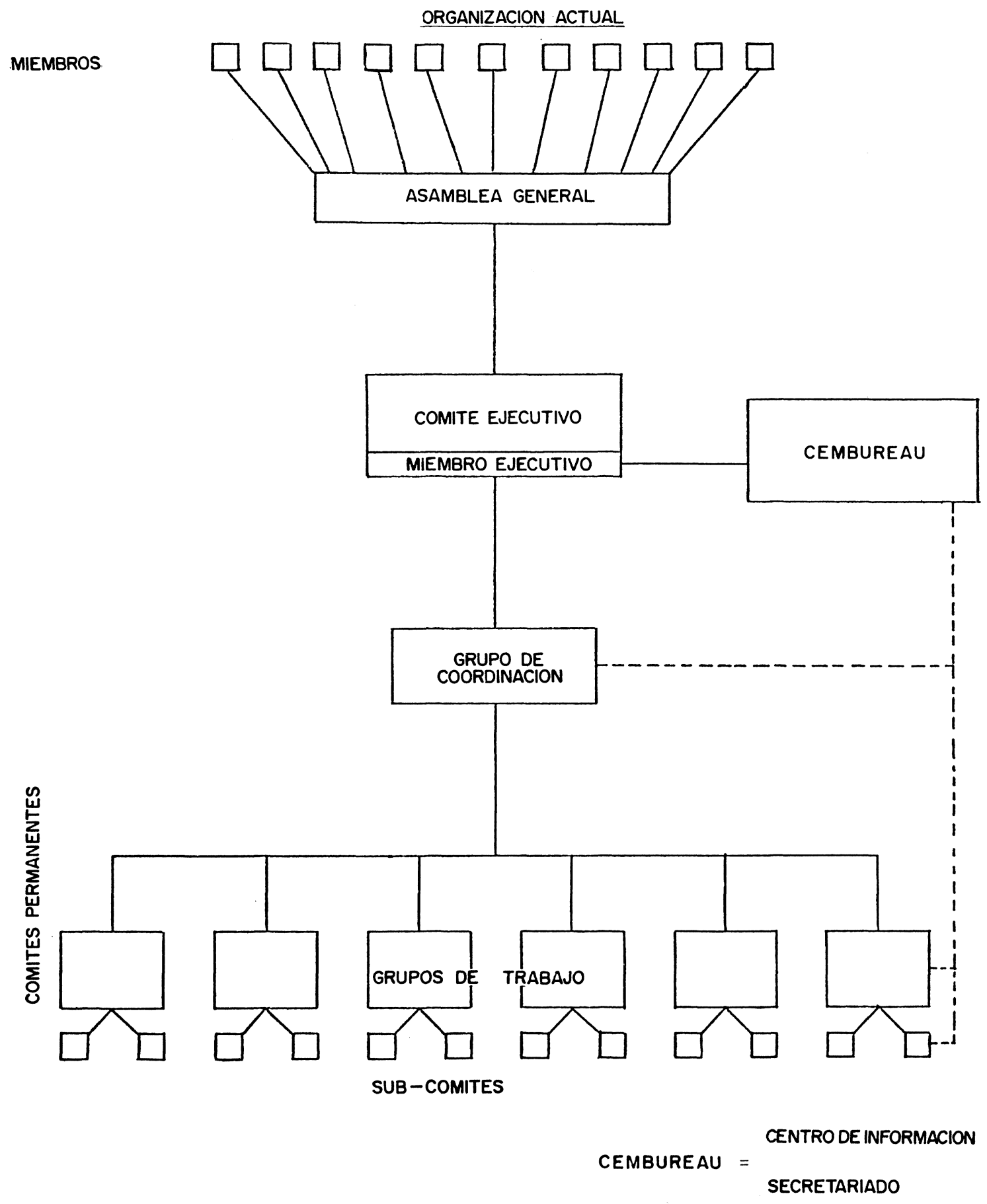




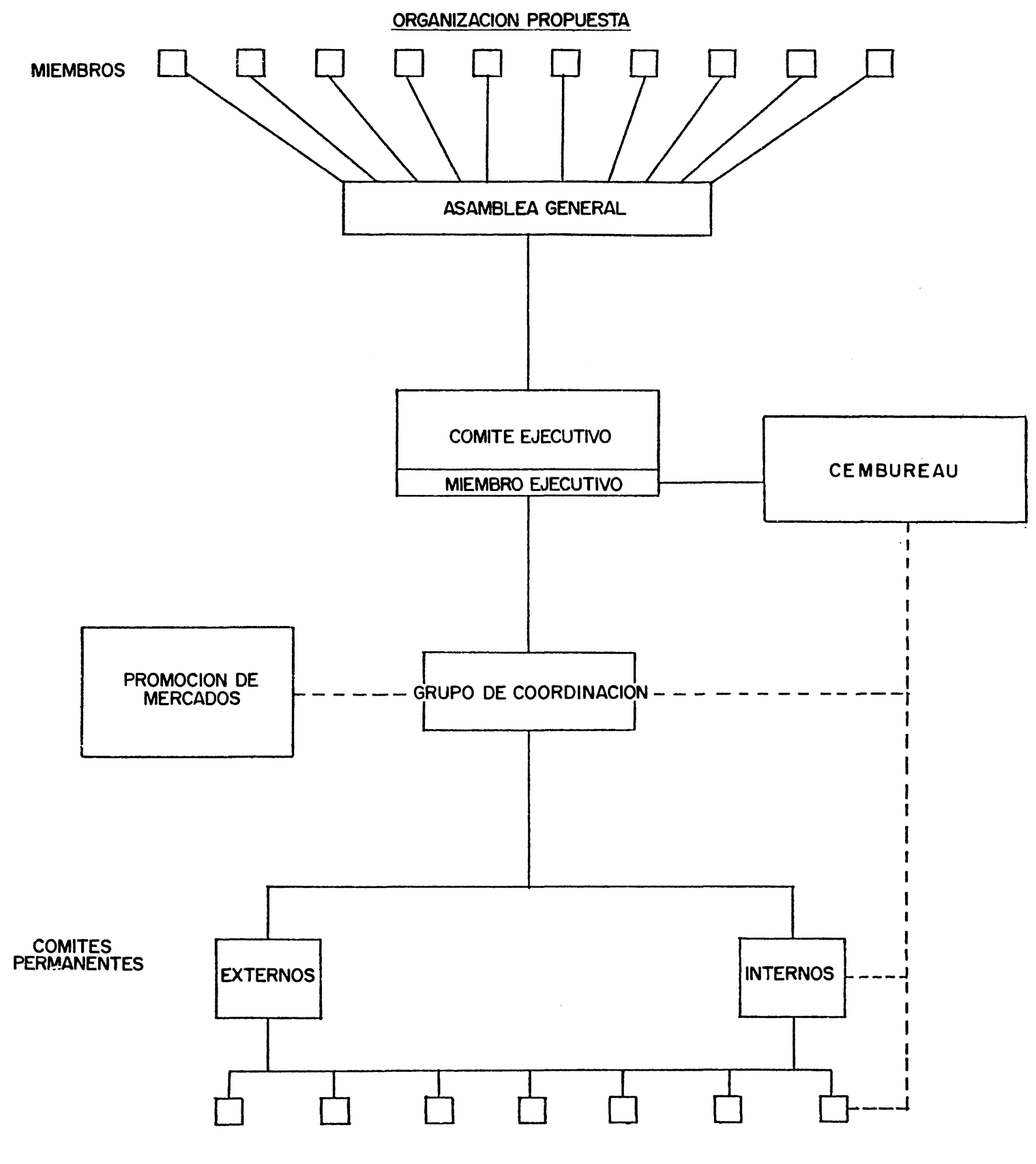

COMITES DE EXPERTOS AD-HOC

CEMBUREAU =

CENTRO DE INFORMACION

SECRETARIADO 


\title{
Relación de los trabajos efectuados por el Sub-Comité de Resistencia Química del CEMBUREAU entre 1962 a 1976
}

\author{
1962 Düsseldorf - Reunión del Sub-Comité
}

Se elaboró un texto sobre los métodos utilizados en los diferentes países, si bien hasta entonces ninguno de dichos métodos se había estandarizado ni era perceptivo.

\section{Londrés - Reunión del Grupo de Trabajo}

Se introdujo el texto formal elaborado por el Sub-Comité y se resaltó la necesidad de distinguir entre la resistencia química intrínseca del cemento y la que podía ser derivada de su impermeabilidad a los agresivos.

\section{Roma - Reunión del Sub-Comité}

Se confirmó la idea inicial de distinción, lo que hizo necesario examinar los métodos utilizados y elegir los que permitieran una mejor separación de las dos variables: resistencia intrínseca y resistencia causada por la impenetrabilidad.

\section{Madrid - Reunión del Grupo de Trabajo}

Se intercambiaron opiniones y se tomó la decisión de examinar de forma crítica y en detalle los métodos conocidos y sus resultados, considerando la experiencia adquirida. Por lo tanto, todos los miembros del Sub-Comité prometieron enviar a los otros miembros, en un plazo máximo de 5 meses, una información completa de sus opiniones sobre el valor de los métodos.

\section{Düsseldorf - Reunión del Sub-Comité}

Los diferentes países miembros informaron sobre los ensayos realizados y se decidió que los mismos debían ser finalizados en la reunión del Grupo de Trabajo.

\section{Viena - Reunión del Grupo de Trabajo}

Se decidió que, de acuerdo con los resultados obtenidos de los diferentes países, ninguno de los métodos conocidos era lo suficientemente bueno para determinar la resistencia a los ataques químicos. Por lo tanto, se recomendó el evitar la implantación de un 
método internacional estándar para este fin. Al propio tiempo se recomendó el entrar en contacto con RILEM con el fin de establecer, si fuera posible, algunas recomendaciones generales sobre la durabilidad del hormigón.

\section{Roma - Reunión del Grupo de Trabajo}

El Sub-Comité finalizó una serie de trabajos, pero sobre los cuales no se ha dado una conclusión hasta la fecha. Se confirmó que los métodos no eran lo suficientemente precisos y que era necesario considerar tres puntos:

a) origen del ataque;

b) los propios métodos de ensayo;

c) la forma de protección a adoptar.

Se decidió distribuir estos temas entre los miembros del Sub-Comité. RILEM aceptó la colaboración propuesta por CEMBUREAU y nombró al Sr. Czernin Presidente del SubComité de RILEM "Ataque Químico" así como intermediario con el CEMBUREAU, como miembro del Sub-Comité y de su Grupo de Trabajo sobre el mismo tema.

\section{Barcelona - Reunión del Sub-Comité}

En 1966 se decidió efectuar un ensayo común basándose en los métodos más importantes con los mismos cementos y en los laboratorios de los diferentes miembros del SubComité. Se aceptaron los métodos siguientes:

Merriman-García de Paredes

CERILH

ASTM

Wittekindt
Koch-Steinegger

Anstett

APCM

Con el fin de conseguir una máxima comparación de los resultados, los participantes se comprometieron a utilizar los métodos sin ninguna modificación; por lo tanto se redactó para cada método un "modus operandi", para las naciones siguientes:

Alemania:

Gran Bretaña:

Francia:

España:

\section{Koch-Steinegger}

\section{$\mathrm{APCM}$}

CERILH

Merriman-García de Paredes

ASTM

Se decidió enviar a todos los participantes, Cementos de Alemania e Italia así como las características de los métodos por parte de los países anteriormente mencionados. 


\section{Estoril - Reunión del Grupo de Trabajo}

Se refirió a la reunión del Sub-Comité en Barcelona. No fue posible presentar ningún resultado dado que los ensayos no se habían finalizado por entonces. Se comentó que el Sub-Comité debería estudiar otros tipos de ataques químicos, independientemente de los del sulfato, pero en vista de la inmensidad de ataques posibles, se decidió que el Sub-Comité debía concentrarse en el ataque de sulfatos.

\section{Atenas - Reunión del Grupo de Trabajo}

Se presentaron ya los resultados de dos ensayos en común, que habían sido indicados el año anterior, pero no pudieron redactarse conclusiones, dado que varios ensayos todavía no habían sido terminados y estaban pendientes de recibir de los miembros.

\section{Dublín - Reunión del Grupo de Trabajo}

Se estableció la conveniencia de continuar con los ensayos en común —si bien únicamente con uno o dos métodos- y hasta ahora los resultados más similares provinieron del Koch-Steinegger y del Merriman-García de Paredes.

\section{Roma - Reunión del Sub-Comité}

Se tomó la decisión de efectuar nuevos ensayos en común con los métodos Koch-Steinegger y Merriman-García de Paredes, utilizando como solución atacante el sulfato sódico, y el probar 9 cementos suministrados por diferentes miembros del Sub-Comité.

\section{Helsinki - Reunión del Grupo de Trabajo}

En vista de las dificultades aparecidas durante el ensayo de Koch-Steinegger con la arena de RILEM-CEMBUREAU, se decidió repetir el ensayo utilizando la misma arena pero sin la parte gruesa, cuyo suministro a todos los participantes debería ser efectuado por Bélgica.

\section{Copenhagen - Reunión del Grupo de Trabajo}

Se dejaron por terminar trabajos y estudios y una vez que los mismos se finalizasen, debía celebrarse una reunión del Sub-Comité con el fin de estudiar los resultados. RILEM acepta la creación de un Comité Mixto. 


\section{9 y1 Zurich - Reunión del Grupo de Trabajo}

Los resultados de los trabajos fueron comprobados y se observó que a pesar de que existía una concordancia entre los laboratorios, los resultados no estaban de acuerdo con la práctica y por ello se decidió parar por algún tiempo los ensayos y resumir las recomendaciones dadas por los diferentes países creando un "Código de buena práctica" para el cual se preparó una encuesta entre los miembros del Sub-Comité.

\section{Barcelona - Reunión del Sub-Comité}

Se discutieron las diferentes contestaciones y se tomó la decisión de preparar un modelo base para el sumario final.

\section{Oslo - Reunión del Grupo de Trabajo}

Pasó a informarse sobre la condición de los trabajos y se decidió que el Sub-Comité debería intentar coordinar, no únicamente lo dicho en los estándars, sino también las recomendaciones dadas como resultado de la experiencia.

\section{3,1974 y 1975}

Se trabaja en la redacción de las recomendaciones y después de reuniones habidas en Düsseldorf, Roma y París, se llega a un acuerdo, presentándose el texto en la última reunión celebrada en París los días 16 y 17 de junio del corriente año; después de haber sido sancionada por la totalidad de los miembros del Grupo de Trabajo, el texto ha pasado a la Secretaría con ligeros retoques y se espera su pronta publicación en el ámbito que crea conveniente el Comité Ejecutivo. 


\section{APENDICE IV}

\section{Recomendaciones para el uso del hormigón en medios agresivos}

Las presentes tablas han sido realizadas teniendo en cuenta los siguientes principios fundamentales: La durabilidad en el sentido más amplio está relacionada con la impermeabilidad del hormigón. Esta impermeabilidad exige que el hormigón esté completamente compactado con un mínimo de poros de aire y agua, lo cual se puede conseguir si se especifica un mínimo adecuado de cemento y una relación agua/cemento máxima, teniendo en cuenta los medios de compactación disponibles y los tipos y las granulometrías de los áridos. Una contribución adicional a la resistencia frente a los agesivos se puede lograr mediante la elección de cementos idóneos.

A medida que la intensidad del ataque químico crece, aumenta el contenido mínimo de cemento y disminuye la relación agua/cemento, hasta el punto en que se considere necesaria una protección superficial. Se toma como punto de partida el caso de una inmersión continua en agua del grifo, en la que no hay problema de ataque químico, pero en la que es preciso un cierto valor mínimo de impermeabilidad para garantizar la durabilidad.

Se considera que los valores del contenido de cemento y de la relación agua/cemento dadas en las tablas proporcionan una base sólida para especificar, admitiéndose una cierta flexibilidad en el caso de tratarse de áridos y medios de compactación particulares. En caso de duda los ensayos de mezclas costituirán siempre una valiosa ayuda para determinar la dosificación dentro del campo de los valores recomendados.

En general, la toma de muestras y los ensayos del agua bastarán para informar acerca de la posibilidad de ataque químico en una obra. Se deberán analizar muestras de terreno si se sospecha que contienen agentes que atacan al hormigón, y si la toma de muestra de agua no es factible, al no estar el terreno suficientemente saturado.

Los valores límites fijados para la calificación de las aguas naturales se aplican a las aguas estancadas y poco fluyentes, en las cuales el ataque no disminuye por su reacción con el hormigón.

El máximo grado de ataque es el factor decisivo para la calificación de un agua, incluso si dicho grado es alcanzado por uno sólo de los valores. En los casos en que dos o más valores se situen en el cuarto superior de los correspondientes intervalos (en el caso del $\mathrm{pH}$ en el cuarto inferior) el ataque resultante se incrementa en un grado.

Para la calificación del terreno el grado de ataque dado en las tablas, es decisivo; decrece al disminuir la permeabilidad del terreno. 
T A B L A 1

Ataque químico del hormigón por aguas y terrenos portadores de sulfatos, calificación y medidas a tomar

\begin{tabular}{|c|c|c|c|c|c|c|c|}
\hline Grados de ataque & Ataque nulo & \multicolumn{3}{|c|}{ Ataque débil } & A taque moderado & Ataque fuerte & Ataque muy fuerte \\
\hline $\begin{array}{l}\mathrm{Su} \text { 'f a to, mg } \\
\mathrm{SO}^{2-} / l \mathrm{de} \text { agua } \\
\left(\mathrm{mg} \mathrm{SO}^{2-} / \mathrm{kg}\right. \\
\text { de terreno seco } \\
\text { all aire })\end{array}$ & $\left(\begin{array}{c}<200 \\
(<2.000)\end{array}\right.$ & $\begin{array}{l}200-6 \\
200-4\end{array}$ & $\begin{array}{r}(2 . \\
4\end{array}$ & $\begin{array}{l}-6.000) \\
600\end{array}$ & $\begin{array}{c}600-3.000 \\
(6.000-12.000)\end{array}$ & $\begin{array}{l}3.000-6.000 \\
(>12.000)\end{array}$ & $>6.000$ \\
\hline $\begin{array}{l}\text { Tipo de cemento } \\
\text { Relación máxi- } \\
\text { ma a g u a/ce- } \\
\text { miento } \\
\text { Contenido míni- } \\
\text { mo de cemento, } \\
\mathrm{kg} / \mathrm{m}^{3}\end{array}$ & $O C$ & OC & $\begin{array}{l}\text { OC } \\
0,50\end{array}$ & $\begin{array}{l}\text { SRC } \\
0,55\end{array}$ & SRC & SRC & 0,45 \\
\hline $\begin{array}{l}\text { Protección adi- } \\
\text { cional del hor- } \\
\text { migón }\end{array}$ & & & & $10 \mathrm{nec}$ & & & no necesaria \\
\hline
\end{tabular}

OC Cemento Portland ordinario.

SRC Cemento resistente a los sulfatos.

NOTAS

Los cementos resiștentes a los sulfatos son los designados comol tales len las normas de los diferentes países.

Los cementos que contienen puzolanas naturales pueden exigir una relación agua/cemento mayor, y por lo tanto puede ser necearia una mayor que la indicada en la tabla.

TA B L A 2

Ataque químico del hormigón por otros agentes distintos de los sulfatos en aguas $y$ terrenos

Calificación y medidas a tomar

\begin{tabular}{|c|c|c|c|c|c|}
\hline Grados de ataque & Ataque nulo & Ataque débil & Ataque moderado & Ataque fuerte & Ataque muy fuerte \\
\hline \multicolumn{6}{|c|}{$A G \cup A$} \\
\hline $\mathrm{pH}$-valor & $>6,5$ & $6,5-5,5$ & $5,5-4,5$ & $4,5-4,0$ & $<4,0$ \\
\hline $\begin{array}{l}\mathrm{CO}_{2} \text { agresivo } \\
\mathrm{mg}^{\mathrm{CO}_{2} / l}\end{array}$ & $<15$ & $15-30$ & $30-60$ & $60-100$ & $>100$ \\
\hline Amonio $\mathrm{mg} \mathrm{NH}_{4}{ }^{+} / l$ & $<15$ & $15-30$ & $30-60$ & $60-100$ & $>100$ \\
\hline Magnesio $\mathrm{mg} \mathrm{Mg}^{2+} / l$ & $<100$ & $100-300$ & $300-1.500$ & $1.500-3.000$ & $>3.000$ \\
\hline \multicolumn{6}{|c|}{ TERRENO } \\
\hline $\begin{array}{l}\text { Grado de acidez se- } \\
\text { gún Baumann Gully }\end{array}$ & $<20$ & $>20$ & 一 & - & - \\
\hline \multicolumn{6}{|c|}{ MEDIDAS NECESARIAS } \\
\hline Tipo de cemento & \multicolumn{5}{|c|}{ Cemento Pontland ordinario } \\
\hline $\begin{array}{l}\text { Contenido mínimo de } \\
\text { cemento } \mathrm{kg} / \mathrm{m}^{3}\end{array}$ & - & 300 & 330 & 370 & 370 \\
\hline $\begin{array}{l}\text { Relación máxima } \\
\text { agua/cemento }\end{array}$ & - & 0,55 & 0,50 & 0,45 & 0,45 \\
\hline $\begin{array}{l}\text { Protección adiccional } \\
\text { del hormigón }\end{array}$ & \multicolumn{4}{|c|}{ no necesaria } & necesaria \\
\hline
\end{tabular}




\section{O LOQU IO}

1. Lo abrió e inició el Presidente, Sr. ARREDONDO, para aclarar algo de lo expuesto por el Sr. BALAGUER, así como un inciso hecho por el Sr. DUTRON en su pasada conferencia, en relación con la ausencia de España en las últimas reuniones del CEN. Puntualizó que quería dejar bien sentado que no había sido debida a descuido, sino tal vez a una accidental falta de coordinación entre organismos españoles. El CEN -dijo-, no se entiende sino con un solo organismo en cada país, que en nuestro caso es el IRANOR. Y puede haber habido ocasiones en que las comunicaciones no hayan llegado, o no se hayan recibido a tiempo. Por ello se han tomado medidas oportunas para procurar evitar que esto ocurra, habiéndonos puesto de acuerdo con el Sr. DUTRON para estar informados al día de las reuniones.

Esto es importante - dijó-, porque las normas del CEN van a ser obligatorias para los países del MCE (Mercado Común Europeo). Por ahora no somos miembros del mismo, pero algún día lo seremos, y podremos encontrarnos entonces con la sorpresa desagradable de que tendremos que adoptar obligatoriamente unas normas en cuya elaboración no hemos intervenido. Creo, por lo tanto -añadió-, que debemos estar presentes en las reuniones, por lo que pueda suceder de cara al futuro.

El Sr. BALAGUER, abundando en esa idea, dijo que en España se hacía en mayor o menor medida toda clase de cementos; que todos los países del MCE tenían sus preferencias por unos u otros; y que por ello teníamos la suerte de que al defender los suyos, siempre defendían alguno de los nuestros. Por ejemplo —añadió-, Inglaterra defiende el cemento portland "puro", hasta el punto de que para obras en el subsuelo de Londres, en el que abundan las piritas y se producen ataques fuertes del hormigón por sulfatos - lo mismo que en Oslo-, utiliza cemento del tipo P-Y, pero no siderúrgico; Bélgica produce y utiliza aproximadamente mitad y mitad de éstos y de portland; Francia es el país de los cementos de mezcla o con constituyentes secundarios - puzolanas, cenizas volantes...-, con indicación de los que en cada caso contienen; Alemania se desentiende de las puzolanas porque produce 4 millones de toneladas de cenizas volantes que, utilizadas como tales, incrementan en igual medida la producción de cemento, cosa que no interesa en un mercado que, como el suyo, está ya al borde de su capacidad de absorción; y en cambio, Italia es un defensor acérrimo de los cementos puzolánicos —porque tiene las mejores puzolanas del mundo-, hasta tal extremo que desde una famosa cantera cercana a Roma se envía puzolana hasta Milán, adonde llega a un precio superior al del clínker, pese a lo cual la utilizan por su fe en ella. Así, pues — concluyó-, al defender cualquiera de estos países sus cementos preferidos, defienden algunos de los nuestros.

2. El Sr. GASPAR intervinó después para decir que hacer bien una norma cuesta bastante dinero, pues exige la intervención de personas expertas en la materia y la realización de unos trabajos experimentales, antes de cubrir todas las etapas que había mencionado el Sr. BALAGUER en su ponencia, hasta el punto de que la elaboración de una norma ASTM costaba en 1965 del orden de los 10.000 dólares. En algunos países -añadió-, es mucho más barato, pues se limitan a traducir y publicar las normas de otros. En ese sentido preguntó qué es lo que se hacía en el sector cementero para hacer una norma del tipo de las de la ISO. 
El Sr. BALAGUER respondió que realmente no podía contestar a ello. Que intervino en la primera redacción del PCCH-61, la cual había llevado siete años de discusiones, ya que fue en la época en que se adoptó la norma ISO-RILEM-CEMBUREAU, pasando del Pliego de 1930, con su mortero de consistencia de tierra húmeda, al mortero plástico, y de unos tipos de probetas y de ensayos a otros. Y que el Pliego preparado por una Comisión pasó después al Ministerio de Obras Públicas, que aceptó, modificó o suprimió lo que le pareció bien; por lo cual no se dió esa tramitación ni esas etapas que se suelen dar en el ámbito internacional.

Por otra parte -continuó el Sr. BALAGUER-, en estos aspectos se parte del principio de que cada país tiene el derecho de hacer lo que mejor le parezca, y que en ese sentido se podía apreciar en una de las tablas con que había acompañado su exposición, relativa a condiciones del hormigón para resistir el ataque de los sulfatos, una observación que, en principio, podía parecer absurda: "Los cementos resistentes a sulfatos son los designados como tales en las normas de los diferentes países". Pero que tenía que ser así, porque para considerar un cemento como resistente a sulfatos, distintos países establecían criterios diferentes. $\mathrm{C}_{3} \mathrm{~A} \%$ máximo $=0(\mathrm{~A} / \mathrm{F}=0,64), \quad$ o $\mathrm{C}_{3} \mathrm{~A} \%$ máximo $=3$, ó $\mathrm{C}_{3} \mathrm{~A} \%$ máximo $=5$; y que el CEMBUREAU no podía implantar uno de estos $\mathrm{u}$ otros valores, porque ello supondría una injerencia en las normas domésticas de la mayoría de ellos.

3. El Sr. GASPAR formuló después otra pregunta en relación con lo dicho por el Sr. ARREDONDO acerca de la necesidad de participación española en la preparación de normas de ámbito europeo por parte del CEN ¿se tienen en cuenta las "observaciones" del CEN en concepto de observador? Porque en caso negativo no valdría la pena asistir.

El Sr. BALAGUER contestó que era bastante lógico que España, como Suiza o Noruega, que no forman parte del MCE, asistieran de vez en cuando como simples observadores, ya que no tienen mucho más que hacer. Porque, como había dicho antes —continuó el Sr. BALAGUER-, un proyecto de norma es aceptado por el CEN si, cumpliendo con una serie de requisitos, se decide por votación de sus miembros de pleno derecho; y que en tal sentido, de no serlo y no poder votar, en efecto tanto daba no asistir.

El Sr. GASPAR añadió que había asistido a alguna de estas reuniones y que las observaciones que habían sido hechas por España habían sido tenidas en cuenta y recogidas en los documentos que se distribuyen después. Y hasta tal punto - dijo-, que algunas veces se había podido frenar lo que pudiera llamarse apetencia de determinados países por defender a ultranza sus cementos preferidos, mediante unas acciones que requerían un tacto especial.

Añadió finalmente que tal vez el Sr. CALLEJA pudiera decir si opinaba lo mismo, y hacer alguna aclaración al respecto.

En efecto, el Sr. CALLEJA dijo que recordaba además otra asistencia del Sr. SORIA y otra u otras dos suyas a las reuniones del CEN. Que, a su entender, al menos en éstas suyas, que fueron de las primeras, se asistía por parte de España a título de observador, sin voz ni voto. Pero que, sin embargo —añadió-, al cumplimentar por escrito los documentos que a veces llegan por una $u$ otra vía solicitando opiniones, sobre todo en los últimos tiempos, se había observado que, efectivamente, cabía la satisfacción de comprobar que tales opiniones eran tomadas en consideración de forma prácticamente total.

Por otra parte y matizando lo dicho por el Sr. BALAGUER en el sentido de que la defensa de cualquier tipo de cemento por cualquier país nos favorecía, pues fuera cual fuese el cemento defendido éste estaba incluido en muestras actuales, el Sr. CALLEJA dijo que "la recíproca era igualmente cierta", y que la actitud de España, incluso representada por un observador sin voz ni voto, había ayudado a veces a determinados países en la defensa de sus cementos — por ejemplo, a Italia con los cementos puzolánicos-, defen- 
diendo también, e indirectamente, los correspondientes cementos españoles. Recordó anecdóticamente al respecto que en una de las reuniones del CEN, en que él se encontraba situado enfrente de los italianos y al lado de los franceses - con belgas y alemanes en otro extremo e ingleses cerrando el cuadro-, se produjo una situación curiosa: los italianos defendían enconadamente sus cementos puzolánicos, y los belgas no menos denodadamente, sus siderúrgicos, siendo apoyados por los alemanes; los franceses ocupaban una posición pro-italiana, bien que defendiendo, no puzolana natural, sino sus cenizas volantes. Y los ingleses, con los que normal y lógicamente no debía de ir la cosa, por no afectarles en lo más mínimo - prosiguió el Sr. CALLEJA - tomaron partido en favor de belgas y alemanes. $Y$ en aquella reunión, en la que no tenía voz —continuó diciendo-, escribí mis argumentos con letras grandes en una hoja de papel, que puse de forma que los italianos de enfrente pudieran leer, dándolos también a conocer verbalmente a los franceses de al lado. Unos y otros me instaron —añadió-, a que interviniese con aquellos argumentos, tal vez pensando que así tendrían en caso necesario un voto más a su favor, pero hube de decirles que yo no podía intervenir ni votar, y que intervinieran ellos, como así lo hicieron.

Lo que sí podemos hacer siempre - concluyó el Sr. CALLEJA-, es responder por escrito a los documentos que nos manden y nos lleguen por uno $u$ otro conducto, en la seguridad de que nuestras respuestas son consideradas en la medida en que en cada circunstancia estimen oportuno. En todo caso, en el futuro parece deseable participar en las reuniones del CEN, no sólo con la presencia, sino también con voz y voto.

4. Finalmente el Sr. GASPAR dijo que, precisamente en el día de la fecha estaba teniendo lugar una reunión del Grupo de Trabajo N. ${ }^{\circ} 4$ del CEN en Bruselas, para tratar del método de determinación del contenido de cenizas volantes en los cementos correspondientes, tema del máximo interés para nosotros, ya que antes de 1980 tendremos una producción anual de cenizas superior a los seis millones de toneladas, a las cuales habrá que buscar aplicación. Muchas hay que sirven, y según la central de origen tienen una regularidad de calidad muy grande y fiable -añadió-; hay fábricas que las estudian desde hace tiempo y las utilizan con éxito, como nos consta. Por todo lo cual - concluyó-, este tema de las cenizas volantes, al igual, que otros que se tratan o puedan tratarse en el CEN, son de gran interés para todo el sector cementero, por lo que considera que nuestra presencia activa en dicho organismo es indispensable.

Y no habiendo más preguntas sobre el tema, el Presidente Sr. ARREDONDO, dió las gracias al Sr. BALAGUER por su exposición, y a los participantes en el Coloquio por su intervención en el mismo, dándolo por concluido. 